\title{
Cognitive Radio Networks- Spectrum Sensing
}

\author{
${ }^{1}$ M. Vinod Kumar Reddy, ${ }^{2}$ K.Arun Kumar \\ ${ }^{1,2}$ Department of ECE, CVR College of Engineering, Ibrahimpatnam, Hyderabad, India \\ Email: ${ }^{1}$ vinodreddy.488@gmail.com, arun.katkoori@gmail.com
}

\begin{abstract}
Cognitive radio plays an important role in wireless communication. The spectrum has become a limited resource by ever increasing demand for wireless communications. For developing of new applications and services the existing spectrum allocation system creating a major problem. Cognitive radios are better solution to the problem of overcrowed and inefficient licensed spectrum. The sensing spectrum is the major key role in cognitive radio to find the free spectrum and favorable circumstances to use the unused frequency bands without causing intervention to the existing primary users(PUs).
\end{abstract}

Index terms - Cognitive radio, spectrum sensing, energy detection, primary user, secondary user.

\section{INTRODUCTION}

Wireless communications can be regarded as the most significant and important development in a modern society. Federal Communications Commission (FCC) found that spectrum access is more significant problem than physical scarcity of spectrum. With many developments in the area of wireless communication technologies already being employed Multimedia Broadcast and Multicast Services (MBMS) demand has tremendously increased and with the standardization of MBMS it has gained significant interest in the market[2]. Because It needs high bandwidth, storage capacity and some applications pose tight delay constraints, so it is necessary to optimize the utilization of spectrum is felt all the more.

Cognitive radio arises to be a solution to spectral crowding problem by introducing the opportunistic usage of frequency bands that are not highly occupied by licensed users since they cannot be utilized by users other than the license owners at the moment It is a system that senses its operational electromagnetic environment and can dynamically and automatically adjust its radio operating parameters to modify system operation, such as mitigate interference, enhance throughput facilitate interoperability, access secondary markets. Moreover, recent calculations by the Federal Communications Commission (FCC) show that the spectrum utilization in the $0-6 \mathrm{GHz}$ band varies from 15 to $85 \%$ based on time, frequency and geographical area as shown in Fig. 1. These observations inspire the development of the cognitive radio (CR) and to modify the current static spectrum access policies accordingly in order to overcome the spectrum sacristy and underutilization problems.[4]

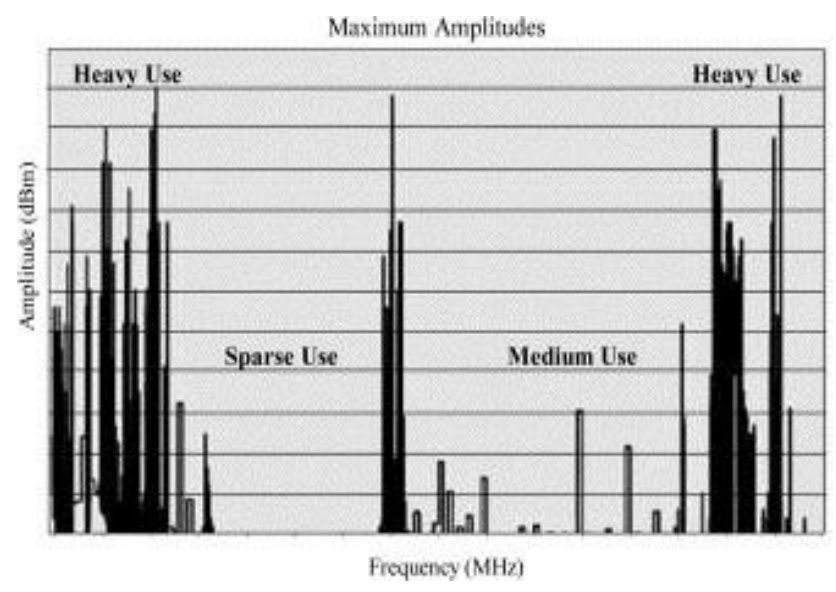

Figure 1: Spectrum Utilization

\section{Cognitive RADIO}

Cognitive radio is the technology which enables next generation communication networks, also known as dynamic spectrum access networks, to utilize the spectrum more efficiently in an opportunistic fashion without interfering with licensed users (primary users).

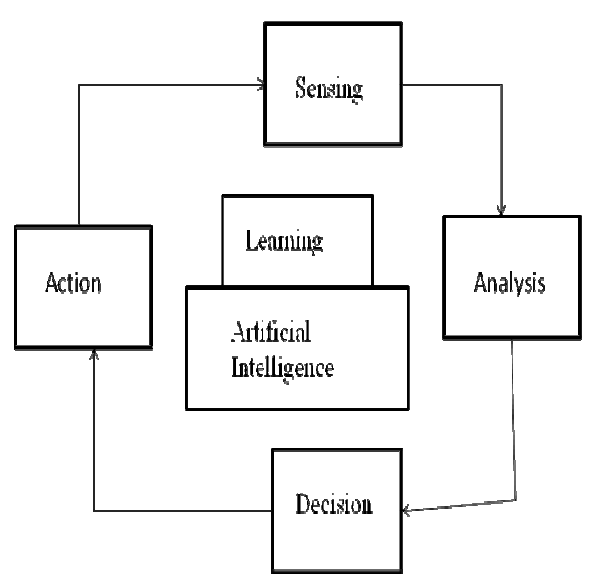

Figure 2: Cognitive Process

A basic cognitive process consists of following three basic tasks:
i. $\quad$ Sensing the spectrum
ii. Analyzing the Spectrum
iii. Decision Making of Spectrum

\section{A. Sensing the Spectrum:}

Spectrum sensing is the ability to measure, sense and be aware of the parameters related to the radio channel 
characteristics, availability of spectrum and transmit power, interference and noise, radio's operating environment, user requirements and applications, available networks (infrastructures) and nodes, local policies and other operating restrictions. It is done across Frequency, Time, Geographical Space, Code and Phase.

\section{B. Analyzing the Spectrum:}

Spectrum Analysis is based on spectrum sensing which is analyzing the situation of several factors in the external and internal radio environment (such as radio frequency spectrum use by neighboring devices, user behavior and network state) and finding the optimal communication protocol and changing frequency or channel accordingly[6]. It is also known as channel estimation.

\section{Decision Making of Spectrum:}

Spectrum Decision Making calls for reconfiguration for the channel and protocol required for constantly adapting to mobile changing environments and adjustment of output power or even alteration of transmission parameters (such as modulation formats (e.g. low to high order QAM), variable symbol rates, different channel coding schemes) and characteristics by the Cognitive radio devices. CR should be able to use multiple antennas for interference nulling, capacity increase or range extension.

\subsection{Characteristics OF Cognitive Radio}

\section{A. Cognitive capability:}

Which is the ability to acquire the radio parameters from its surroundings.CR should be able to determine the frequency occupancy by identifying the spectrum holes (or spectrum opportunities). The spectrum hole is defined as the frequency bands which are allocated but not utilized in some location and at some times by the licensed system as given in Fig 3. Moreover, depending on the system, CR might have information about the modulation and coding as well as the relocation of the licensed system devices. $C$.

\section{B. Reconfigurability:}

Which is the ability to rapidly adapt the transmit parameters, i.e. operating frequency, modulation and coding, transmit power and communication technology, according to the radio environment in order to achieve the optimal performance

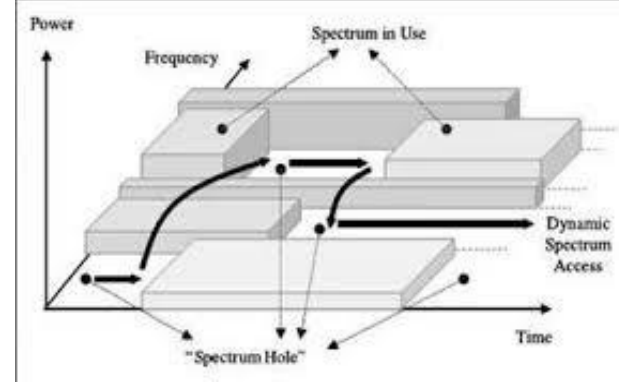

Figure 3: Spectrum Opportunities

\subsection{FunCtions of Cognitive Radio}

The CR network has two main components: the primary network and the secondary one. The primary network, also referred to as licensed network, has a license to operate in a certain frequency band. It consists of primary users (PUs) with/without primary base stations (BSs). PUs is generally not equipped with any CR functions [8]. On the other side, the secondary network is able to access the licensed spectrum without affecting the primary network transmission. The secondary network is composed of secondary users (SUs) with/without secondary BS. Additionally, spectrum broker can be used to enable efficient and fair spectrum sharing between multiple secondary networks coexist in the same frequency band.

To support this type of spectrum sharing between the primary and cognitive networks, and to guarantee efficient usage of the resources in both networks, CR is required to perform the following four functions

\section{A. A. Spectrum Sensing:}

By this function, in order to identify the PUs activity the $\mathrm{CR}$ guides its radio environment Based on the sensing information, CR can determine the available spectrum holes that can be used for the CR transmission in a particular time, frequency, and location. Furthermore, the CR need to keep sensing the frequency spectrum during the $\mathrm{CR}$ transmission to avoid interfering with reappeared PUs.

\section{B. Spectrum Decision:}

This function analyzes the information from the spectrum sensing phase. The characteristics of the detected spectrum holes, the probability of the PU appearance, and the possible sensing errors should be considered before making the spectrum access decision [7]. Once the appropriate band is selected, the $\mathrm{CR}$ has to optimize the available system resources in order to achieve the required objective.

\section{Spectrum Sharing:}

This function chooses the appropriate MAC protocol to access the spectrum holes. By the MAC protocol, fair spectrum sharing between the different SUs can be guaranteed. Additionally, coordination between nodes can be achieved in order to avoid the collision with PUs as well as other SUs.

\section{Spectrum Mobility:}

Also called spectrum handover and by this function, CR is able to change the operating band in order to avoid a detected PU activity. Additionally, the CR can perform the spectrum handover in order to improve the secondary network performance by transmitting in another spectrum hole with better condition [6]. The protocol parameters at the different levels should be adapted according to the new operating band.

\section{SPECTRUM SENSING}

Spectrum sensing is universally recognized as main enabler for cognitive radio. The actual meaning of 
spectrum sensing is to provide awareness of the radio environment gives indication on the availability of transmission opportunities, gives indication on who is occupying the channel and gives indication on the quality of channel itself.

\subsection{BASICS-COGNITIVE RADIO -SPECTRUM SENSING}

There are various of considerations in Frequency spectrum sensing occupancy. They are

\section{A. Continuous Spectrum Sensing:}

In the cognitive radio, we have to continuously sense the spectrum occupancy. Generally a cognitive radio employs the spectrum on a non-intervention basis to the primary user. Correspondingly, it is required for the cognitive radio system to continuously sense the spectrum in case the actual primary user returns.

\section{B. Monitoring for alternative Empty Spectrum:}

The cognitive radio system must have alternate spectrum available to which it can change should the right appear if the existing primary user returns to original spectrum which is being used.

\section{Monitoring the type Of Transmission:}

Whatever the type of transmission being received is required to sense in cognitive radios and cognitive radio system be able to find the type of transmission used by the actual primary user so that specious transmissions and interference are ignored as well as transmissions made by the cognitive radio system itself[4].

There are number of ways in which cognitive radios are able to perform frequency spectrum sensing. The ways in which cognitive radio frequency spectrum sensing can be performed in any one of the following methods:

\section{i. Non-cooperative:}

In this, frequency spectrum sensing occurs, when a CR system acts on its own. The cognitive radio system will configure its own according to the signals it can find the information with which it is preloaded.

\section{ii. Cooperative:}

In this, sensing will be initiated by a number of different radio systems within the cognitive radio network. Typically a central station will receive reports of signals from different radio stations in the network and adjust overall cognitive radio network to suit.

The problems of unnecessary interference is reduces in cognitive radio cooperation where a single cognitive radio cannot hear a primary user because of issues such as changing from primary user, but second primary user acts as a receiver may be able to hear both the primary and the signal from the cognitive radio system.

\section{SPECTRUM SENSING MethodS}

In this paper we discuss about some of the spectrum sensing techniques.

They are:

\section{A. Energy Detection}

It is most common way of spectrum sensing because of its low computational and implementation complexities [6]. This technique also known as radiometry and it is more generic as receivers do not require any knowledge on the Primary signal energy. It consists in extracting as a feature the incoming signal power, normalized by the noise power.

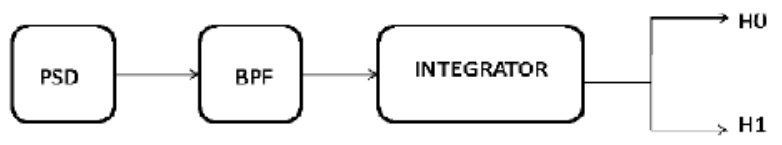

Figure 4: Block Diagram of Energy Detection

Where H0 Absence of user

H1 Presence of user

Block diagram for the energy detection technique is shown in Figure 4. In this, First the power spectral density of the signal is calculated and this is passed through band pass filter of the bandwidth $\mathrm{W}$ and is integrated over time interval. The output from integrator is then compared with the predefined threshold value. This comparison is used to find the existence or absence of the primary user and this value can set to be fixed or variable based on the channel conditions.

$\mathrm{y}(\mathrm{k})=\mathrm{n}(\mathrm{k}) \ldots \ldots \ldots \ldots \mathrm{H} 0$
$\mathrm{y}(\mathrm{k})=\mathrm{h} * \mathrm{~s}(\mathrm{k})+\mathrm{n}(\mathrm{k}) \ldots \ldots \mathrm{H} 1$

$\mathrm{y}(\mathrm{k})$ is the sample to be analyzed at each instant $\mathrm{k}$ and $\mathrm{n}$ (k) is the noise of variance $\sigma^{2}$. Let $\mathrm{y}(\mathrm{k})$ be a sequence of received samples $\mathrm{k} \varepsilon\{1,2 \ldots \mathrm{N}\}$ at the signal detector, then a decision rule can be stated as, $\mathrm{H} 0 \ldots \ldots$ if $\varepsilon>\mathrm{v}$ and $\mathrm{H} 1 \ldots \ldots$ if $\varepsilon<\mathrm{v}$

Where $\varepsilon=\mathrm{E}|\mathrm{y}(\mathrm{k})|_{2}$ the estimated energy of the received signal and $\mathrm{v}$ is chosen to be the noise variance $\sigma_{2}$. Any how the energy detection has the following disadvantages

i) The Sensing time taken to achieve a given probability of detection may be more.

ii) The detection performance is subject to the uncertainty of noise power.

iii) The energy Detection cannot be used to detect spread spectrum signals.

\section{B. Matched Filter Detection:}

A matched filter is a linear filter which increases the output SNR for an inclined input signal [6]. When primary user's a priori knowledge is known, the Matched filter detection is applied. In matched filter operation the unknown signal is convolved with the filter's impulse response which is time shifted and mirror version of the actual primary user signal. Mathematically, matched filter operation can be expressed as:

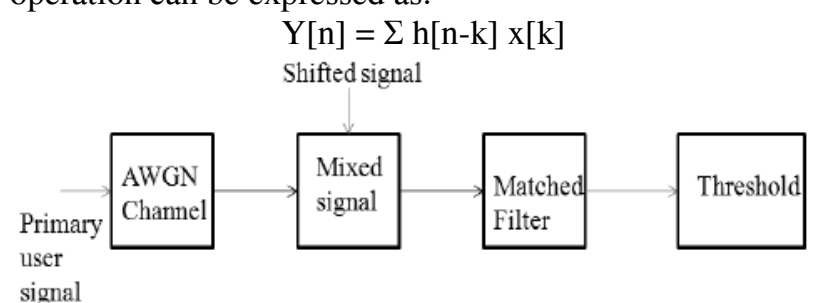

Figure 5: Block diagram of matched filter detection 
From the above block diagram, the primary user signal is convolved with time shifted version of the prior noon signal. The final output of the filter is compared with the threshold will determine the primary user presence [7].

\section{Cooperative Sensing:}

It is used for detecting primary user effectively in high noisy environment, where multiple secondary users make a global decision in relation to primary user[8]. this method is a solution to problems that arise on spectrum sensing due to noise uncertainty, fading and shadowing.

Cooperative sensing techniques:

- Centralized Coordinated

- Decentralized Coordinated

- Decentralized Uncoordinated

Necessisity of cooperative sensing in cognitive radio networks

- Plummeting sensitivity requirements: channel deteriorations like multipath fading, shadowing and building penetration losses, impose high sensitivity requirements inherently limited by power and cost requirements.

- Employing aid between nodes can immensely reduce the sensitivity requirements up to -25 $\mathrm{dBm}$.

- Reduction in sensitivity threshold can be obtained by using cooperation technique.

From above spectrum sensing methods we find some disadvantages like high sensing time taken to achieve a given probability and difficult to differentiate primary user and secondary use signals in energy detection sensing technique, matched filter detection requires prior knowledge of every primary signal and separate receiver for every type of primary user is needed, traffic overhead in cooperative sensing technique. To solve all these problems adaptive spectrum sensing is used.

\section{Adaptive Spectrum Sensing:}

It is a combination of both single energy sensing and cooperative energy sensing. In this technique SNR is compared with threshold value. If SNR is more single energy sensing is used and if threshold is more cooperative energy sensing is used[6][9].

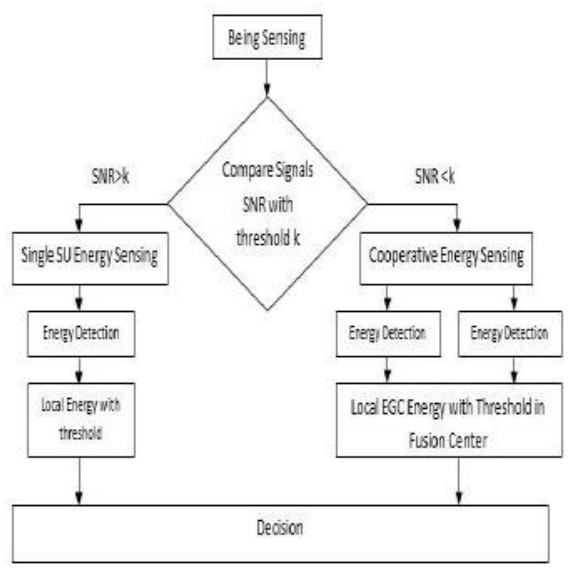

Figure 6: Representation of adaptive spectrum Sensing

\section{Conclusions}

The spectrum is a most valuable resource in wireless communication systems and it has been a major research area from last several decades. Cognitive radio is a promising technology which enables spaces. Considering the challenges raised by cognitive radio systems, the use of spectrum sensing methods appears as an essential need to achieve accurate results in terms of efficient use of available spectrum and limited interference with the licensed primary users. In this method, the signal detection is performed by comparing the output of energy detector with a given threshold value. Adaptive spectrum sensing method according to the Secondary users SNR status by selecting both single sensing and cooperative sensing and confirm performance improvement. We also said about importance of cooperative and adaptive cooperative sensing.

\section{REFERENCES}

[1] Danijela Cabric, Artem Tkachenko, Robert W. Brodersen,"Spectrum Sensing Measurements of Pilot, Energy, and Col-laborative Detection", Military Communication Conference

[2] Weifang Wang, "Spectrum Sensing for Cognitive radio'Third International Symposium on Intelligent Information Technology Application Workshops, pp: 410-412, $2009 .$.

[3] A.Rahim Biswas, Tuncer Can Aysal, SithamparanathanKandeepan,DzmitryKliazovich,

RadoslawPiesiewicz, "Cooperative Shared

Spectrum Sensing for Dynamic Cognitive Radio

Networks",IEEEInternatiional Conference on Communications (ICC), pp. 1-5,June 2009.

[4] Yi Zheng, XianzhongXie, Lili Yang, "Cooperative SpectrumSensing Based on Blind Source Separation for Cognitive Radio",International Conference on Future Information Network(ICFIN),pp. 398-402, Oct 2009.

[5] Hang Su, Xi Zhang, "Energy-Efficient Spectrum Sensing for Cognitive Radio Networks", IEEE ICC, 2010.

[6] T. Yucek, H. Arslan, "A survey of spectrum sensing algorithms for cognitive radio applications", IEEE Communications Surveys Tutorials, vol. 11, no. 1, pp. 116130, 2009

[7] W. Yue and B. Zheng, Spectrum sensing algorithms for primary detection based on reliability in cognitive radio systems, Journal of Compute and Electrical Engineering, vol.36, no.3, pp.469-479,2010.

[8] J. Shen, S. Liu, L. Zeng, G. Xie, J. Gao, and Y. Liu, "Optimization of cooperative spectrum sensing in cognitive radio network," Communications, IET, vol. 3, pp. 11701178, 2009.

[9] Z. Wei, R. K. Mallik, and K. Ben Letaief, "Cooperative Spectrum Sensing Optimization in Cognitive Radio Networks," in Communications, 2008. ICC '08. IEEE International Conference on, 2008, pp. 3411-3415. 